\section{New PAMs for Cas}

The ease of use and robustness of the CRISPR-Cas9 system has made it the go-to tool for genome engineering. But one drawback of the Cas9 endonucleases currently in use is their dependence on the presence of a so-called protospacer adjacent motif (PAM), a set of three or more nucleotides immediately following the DNA sequence targeted by the CRISPR guide RNA (gRNA). For example, the PAM of Streptococcus pyogenes Cas9 (SpCas9), NGG, should occur only once in every eight base pairs of random DNA; however, because genome sequences are not entirely random, it can be challenging to find targets with the PAM sequence in the human genome that are amenable to editing. As David Liu of Harvard University (Cambridge, MA, USA) puts it, "Researchers can be surprised by how restrictive the PAM requirement turns out to be in practice." This has spurred the search for CRISPR-Cas9 variants with different PAM requirements.

In a paper recently published in Nature, Kleinstiver et al. ${ }^{1}$ present variants of SpCas9 with altered PAM specificities. To find these variants, they use a bacterial selection system in which survival of the bacterium depends on Cas9 cleavage of an inducible gene encoding the toxin ccdB. Guided by structural information, the authors prepared a selection library, by randomly mutagenizing the PAM-interacting domains of either the wild-type SpCas9 or SpCas9 variants, each of which contained rationally designed mutations suggested by a Cas 9 crystal structure $^{2}$, and screened the library against target sequences with either an NGA or an NGCG PAM sequence. After selection, sequencing showed that for each PAM recognized by an SpCas9, certain SpCas9 mutations occurred with substantially higher frequencies than others, and that these alterations were physically close to the PAM in the crystal structure. The authors then tested various combinations of these mutations to identify optimal sets of changes that altered PAM recognition. "The best combinations of mutations did not come directly out of our screen, which is not surprising, given the vast combinatorial space," says Keith Joung, the senior author of the study.

To identify the PAM preferences of their engineered SpCas9 variants, the authors performed a second bacteria-based selection.
In this experiment, plasmids that contained a PAM sequence recognized by a given Cas9 variant were cleaved and depleted from a library that contained six randomized bases next to the gRNA binding sequence. Comparison of the PAM-sequence abundance in the control and Cas9-expressing samples yielded the relative activity of each variant toward all possible PAM sequences. "Performing all the screens in cells was a smart move," says Liu, who was not involved in this study, "as it automatically selects against variants with poor specificity or [ones] that cannot fold in vivo."

The top variants contained three (in the case of NGA) and four (in the case of NGCG) mutations compared with the wildtype protein. The activity of the new Cas9 variants is further modulated by an additional base at the $3^{\prime}$ end of the PAM. For example, one of the variants derived from the screen for an NGA-specific Cas9 preferred "G" as in NGAG to all the other possible bases following NGA. In human cells and zebrafish embryos, the

\section{"The results suggest that one} day it may be possible to construct a comprehensive library of Cas9 endonucleases that work with all possible PAM sequences."

variants showed robust activity recapitulating the specificity pattern observed in bacteria. Using the group's own GUIDE-seq technique ${ }^{3}$, Kleinstiver et al. ${ }^{1}$ also established that the new variants have a genome-wide specificity comparable to wild-type SpCas9 in human cells. Interestingly, one of the mutations found in the initial screen that retained the original NGG PAM preference had a substantially lower offtarget activity, irrespective of whether off-target sites contained the canonical PAM or not.

"One of the key findings of the paper is that Cas9 can be evolved to acquire new properties, although of course we don't yet know which useful new functions can be readily accessed in the sequence space of the Cas9 protein," says Liu. Joung adds, “The mutations presented here are a useful starting point for new engineering enterprises as it is likely that it will, for example, be much easier to go from an NGA PAM to an NAA PAM."

As a proof of principle demonstration, the authors also show that smaller Cas9 endonucleases from two other microorganisms work well in the same selection systems, suggesting the same approaches could be used to engineer Cas9s from other organisms that have different PAM requirements. The authors did not attempt, however, to evolve variants with new specificities for these Cas9 proteins in this work. Nevertheless, the results suggest that one day it may be possible to construct a comprehensive library of Cas9 endonucleases that work with all possible PAM sequences.

The current work should not be seen only as a way to circumvent stringent PAM specificities, as Joung also points out that such specificities can be an advantage. "The very strict requirement of the PAM sequence might be used to facilitate truly single nucleotide variant-specific genome editing of individual alleles in a way that is hard to imagine with other tools," says Joung.

Markus Elsner, Senior Editor

1. Kleinstiver, B.P. et al. Nature doi:10.1038/ nature14592 (22 June 2015).

2. Anders, C., Niewoehner, O., Duerst, A. \& Jinek, M. Nature 513, 569-573 (2014).

3. Tsai, S.Q. et al. Nat. Biotechnol. 33, 187-197 (2015). 